\title{
Metabolomic responses to sublethal contaminant exposure in neonate and adult Daphnia magna
}

Nicole D. Wagner, André J. Simpson, Myrna J. Simpson

\author{
Version Post-print/accepted manuscript \\ Citation Wagner, N. D., Simpson, A. J. and Simpson, M. J. (2017), Metabolomic \\ (published version) responses to sublethal contaminant exposure in neonate and \\ adult Daphnia magna. Environ Toxicol Chem, 36: 938-946. \\ doi:10.1002/etc.3604
}

Publisher's statement This is the peer reviewed version of the following article: Wagner, N. D., Simpson, A. J. and Simpson, M. J. (2017), Metabolomic responses to sublethal contaminant exposure in neonate and adult Daphnia magna. Environ Toxicol Chem, 36: 938-946 which has been published in final form at https://dx.doi.org/10.1002/etc.3604. This article may be used for non-commercial purposes in accordance with Wiley Terms and Conditions for Self-Archiving.

How to cite TSpace items

Always cite the published version, so the author(s) will receive recognition through services that track citation counts, e.g. Scopus. If you need to cite the page number of the author manuscript from TSpace because you cannot access the published version, then cite the TSpace version in addition to the published version using the permanent URI (handle) found on the record page.

This article was made openly accessible by $U$ of $T$ Faculty. Please tell us how this access benefits you. Your story matters. 
1 Running title: Age specific metabolic responses to contaminant exposure

2

3

4

5

6 Corresponding author:

7 Myrna J. Simpson

8 Environmental NMR Centre and Department of Physical and Environmental Sciences

91265 Military Trail, Toronto

10 Ontario Canada

$11 \quad \mathrm{M} 1 \mathrm{C}$ 1A4

$12 \quad 416-287-7234$

13 Email: myrna.simpson@utoronto.ca

14

15

16

17 
20 Metabolomic responses to sub-lethal contaminant exposure in neonate and adult Daphnia

21

22

23

24

25

26 Environmental NMR Centre and Department of Physical and Environmental Sciences,

27 University of Toronto Scarborough. Scarborough, ON, Canada.

$28 *$ Corresponding author Myrna J. Simpson email: myrna.simpson@utoronto.ca 
ABSTRACT

30 The use of consumer products and pharmaceuticals that act as contaminants entering waterways

31 through runoff and wastewater effluents alter aquatic ecosystem health. Traditional toxicological

32 end points may underestimate the toxicity of contaminants as lethal concentrations are often

33 orders of magnitude higher than that found within freshwater ecosystems. While newer

34 techniques examine the metabolic responses of sub-lethal contaminant exposure, there has been no direct comparison with ontogeny in Daphnia. We hypothesize that Daphnia magna will have

distinct metabolic changes to 3 different sub-lethal contaminant exposures caused by differences in the toxic mode of action and ontogeny. To test this hypothesis, we measured the proton nuclear magnetic resonance metabolomic profiles in $D$. magna aged day 0 and 18 after exposure to $28 \%$ of the lethal concentration of $50 \%$ of organisms tested (LC50) of atrazine, propranolol and perfluorooctanesulfonic acid (PFOS) for 48 hours. Principal component analysis revealed significant separation of contaminants from the control daphnids in both neonates and adults exposed to propranolol and PFOS. In contrast, atrazine exposure caused separation from the controls in only the adult D. magna. Propranolol exposure displayed minimal ontogenetic changes in the targeted metabolites. For both atrazine and PFOS exposures ontogeny exhibited unique changes in the targeted metabolites. These results indicate that depending on the contaminant studied, neonates and adults respond uniquely to sub-lethal contaminant exposure.

Keywords ${ }^{1} \mathrm{H}$ NMR, PFOS, Propranolol, Atrazine, Environmental metabolomics 
Anthropogenic activity is increasingly altering aquatic ecosystem health both directly and

52 indirectly through wastewater effluents and changes in land use [1,2]. Contaminants enter

53 aquatic ecosystems through wastewater effluents containing personal care products and

54 pharmaceuticals that remain in treated wastewater due to inefficient removal methods $[3,4]$. Land

55 use changes to agriculture, especially to monoculture, leads to increases in run off into nearby

56 riparian zones ultimately increasing turbidity [5], and contaminants (pesticides) [6] entering

57 rivers and lakes. Upon entering aquatic ecosystems the fate of contaminants depends on both

58 abiotic and biotic factors $[7,8]$; however, the presence of contaminants can potentially disrupt or

59 alter ecosystem dynamics such as changes in biodiversity and nutrient cycling $[9,10]$.

60 Contaminants elicit lethal and sub-lethal responses to many different aquatic organisms

61 including producers such as chlorophytes [11,12], cyanophytes [11], and consumers such as

62 cladocerans [13-15], benthic invertebrates [16,17], and vertebrates [18,19]. The freshwater

63 consumer, Daphnia magna, is an important aquatic species capable of dominating the

64 zooplankton biomass [20] thus controlling both top down and bottom up ecosystem processes

65 [21]. While Daphnia spp. are capable of large biomasses within lentic and pelagic zones they are

66 sensitive to many types of environmental stressors including food quality [22], predation [21],

67 and contaminants [23-25] that can change population dynamics [26], and biogeochemical cycles

$68[10,20]$. Given the ecological importance and the life history (i.e. short life cycle) of D. magna it

69 is often the choice of many toxicity experiments to study and predict the ecological

70 consequences of contaminants in aquatic ecosystems [12,13,23,27,28]. However, traditional

71 toxicity end points such as lethal concentration of 50\% of organisms tested (LC50), may 
underestimate the stress caused by sub-lethal contaminant exposure as lethal concentrations are often magnitudes higher than what is present within aquatic ecosystems [10].

Recently many toxicity studies have shifted their focus from terminal (e.g. death) to more sub-lethal metrics such as growth [23], reproduction [23], and molecular-level responses [29] to gain a better understanding of how contaminants alter the physiology of organisms. An emerging field well suited to study the molecular responses of organisms exposed to contaminants is metabolomics, which investigates how a subset of metabolites (polar or non-polar) are altered as a result of sub-lethal contaminant exposure $[15,30,31]$. The advancement of analytical tools such as mass spectrometry [32] and microprobes for nuclear magnetic resonance (NMR) [33] have increased the sensitivity and decreased the mass requirements for metabolomics, thus improving the feasibility to use small organisms such as Daphnia spp. Traditional acute toxicity tests such as the LC50 use neonate D. magna $[12,13,27]$, whereas metabolomic studies use either adult (greater than 14 days old; $[15,34]$ ) or neonate D. magna $[35,36]$ to determine the changes in the metabolic profile caused by the contaminant exposure. As the field of toxicology transitions from traditional lethal studies to more modern sub-lethal metrics there will become an increasing need to compare whether animals will respond similarly throughout ontogeny. However, to our knowledge no studies have examined if ontogenetic differences in D. magna will alter their metabolic profile to contaminant exposure.

Daphnia spp. 's life history consists of being an r-selected species that emphasizes energy into reproduction [20]. Typically, daphnids have fast growth rates and can reach reproduction at approximately 8 days old under optimal conditions [22]. Once Daphnia spp. begin to reproduce, their energetic demands shift from growth to reproduction, and thus growth rates decline. Energetic demands can be thought of as a hieratical system with prioritizing maintenance, then 
growth and finally reproduction as described in dynamic energy budget theory [37]. These differences in energetic demand can be amplified especially for acute toxicity tests as reproduction is not a continuous energy demand unlike growth [38]. Given the differences in energetic demand between the two life stages of Daphnia spp. in addition to previous research that has shown neonates are more susceptible to toxicity $[39,40]$, a direct comparison between the metabolic profiles of neonate and adult D. magna exposed to contaminants is needed to determine if their profiles respond similarly to contaminant stress.

Here we examine the metabolomic responses between neonate and adult D. magna exposed to 3 different contaminants for 48 hours. Both neonate and adult D. magna were exposed to one concentration of propranolol, atrazine, or perfluorooctanesulfonic acid (PFOS) and their metabolic profiles were measured using ${ }^{1} \mathrm{H}$ NMR. Propranolol is a pharmaceutical used to treat hypertension by acting as a non-specific $\beta$-blocker causing vasodilation. This pharmaceutical enters aquatic ecosystems through inefficient removal during wastewater treatment [23]. Atrazine is a commonly used agricultural herbicide that inhibits the photosystem II in plants, and enters aquatic ecosystems through run off [41]. PFOS is a common consumer product used as a stain repellent on clothing, as well used as a surfactant, and enters aquatic ecosystems through inefficient removal in treating wastewater [12]. Given the difference type of contaminants, we hypothesize D. magna will have distinct metabolic profiles caused by differences in the toxic mode of action (MOA) and ontogeny. We expect neonate D. magna to respond more severely than adult D. magna to contaminant stress because of differences in energy demands for maintenance, growth and reproduction. 
Algae and Daphnia magna Culturing

Raphidocelis subcapitata (Canadian Phycological Culture Centre strain 37) was grown in multiple batch culture jars in Bristol media with a $16: 8 \mathrm{~h}$ light : dark cycle, and a light intensity of approximately $150 \mu \mathrm{mol} / \mathrm{s} / \mathrm{m}$ at $20^{\circ} \mathrm{C}$. Each batch culture was concentrated by centrifugation at $4066 \mathrm{~g}$ for $20 \mathrm{~min}$ and fed to brood mother D. magna ad libitum.

Daphnia magna (Straus) was purchased from Ward Science Canada in 2013 and has since been raised at $20^{\circ} \mathrm{C}$ in municipal de-chlorinated tap (Toronto, Ontario, Canada) water with a hardness of approximately $120 \mathrm{mg} \mathrm{CaCO}_{3} / \mathrm{L}$ and $\mathrm{pH} 8.15$. Animals were maintained in a density of 1 daphnid per $30 \mathrm{~mL}$ water and fed $R$. subcapitata ad libitum daily with a $16: 8 \mathrm{~h}$ light: dark cycle. Water was changed by replacing $50 \%$ of the old water with fresh de-chlorinated tap water to remove excess food and ammonium every other day. In addition to water changes, D. magna were supplemented with $1 \mu \mathrm{g} / \mathrm{L}$ of selenium and cobalamin bi-weekly to ensure animals received adequate essential nutrients not provided by the algae [42].

\section{Contaminant exposure}

Cultured daphnids were used to study the metabolomic changes in both neonate and adult D. magna exposed to sub-lethal concentrations of atrazine, propranolol, and PFOS. We used $D$. magna literature values of acute $48 \mathrm{~h}$ LC50 for both atrazine $(35.5 \mathrm{mg} / \mathrm{L}[13,28])$ and PFOS $(130$ $\mathrm{mg} / \mathrm{L}[12])$ to determine sub-lethal concentrations for our exposures. However, propranolol literature LC50 value (7.7 mg/L [27]) resulted in 100\% mortality, thus 48 h LC50 experiments were conducted using Environment Canada's protocol [42]. Briefly, neonates under 24 h old were removed from brood mother jars and placed in groups of 10 in $200 \mathrm{~mL}$ of de-chlorinated 
tap water with varying concentrations of propranolol and checked for mortality after $48 \mathrm{~h}$ ( $n=5$; Supplemental Data, Figure S1).

For both neonate and adult exposures, we used concentrations consisting of $28 \%$ of the $48 \mathrm{~h} \mathrm{LC50} \mathrm{as} \mathrm{initial} \mathrm{trials} \mathrm{resulted} \mathrm{in} \mathrm{no} \mathrm{mortality.} \mathrm{This} \mathrm{represents} \mathrm{a} \mathrm{nominal} \mathrm{concentration} \mathrm{of} 10$ $\mathrm{mg} / \mathrm{L}$ atrazine (PENSTAL $98.8 \%$ purity Sigma-Aldrich, Oakville, ON), $36 \mathrm{mg} / \mathrm{L}$ PFOS (98\% purity, Sigma-Aldrich, Oakville, ON), and $0.67 \mathrm{mg} / \mathrm{L}$ propranolol (propranolol hydrochloride, $<$ $99 \%$ purity, Sigma-Aldrich, Oakville, ON). Both PFOS and propranolol were hydrophilic enough to be dissolved directly into the de-chlorinated tap water; however a solvent carrier of $0.15 \%$ acetone was needed to make stock solutions of atrazine. The final concentration of acetone was $0.03 \%$ used in the neonate and adult exposure experiments.

\section{Neonate specific experimental design}

Stock concentrations of each contaminant were made in de-chlorinated tap water. An aliquot of the contaminant was added to replicate ( $n=8$ per contaminant exposure) $2 \mathrm{~L}$ glass jars and filled with additional de-chlorinated tap water to a final volume of $2 \mathrm{~L}$ to obtain the nominal concentration of each contaminant $(0.67 \mathrm{mg} / \mathrm{L}$ propranolol, $10 \mathrm{mg} / \mathrm{L}$ atrazine, $36 \mathrm{mg} / \mathrm{L}$ PFOS; $n$

$=8$ per contaminant exposure). This procedure was repeated for all the treatments (propranolol, atrazine, and PFOS). Additional replicates $(n=8)$ of $2 \mathrm{~L}$ glass jars were filled with dechlorinated tap water as a control treatment for the propranolol and PFOS treatments, whereas the atrazine treatment controls $(n=8)$ contained $0.03 \%$ acetone as a solvent control. Daphnia magna neonates ( $<24 \mathrm{~h}$ old) were removed from the brood mother cultures and pooled into a container then placed into experimental glass jars with the density of 1 neonate per $30 \mathrm{~mL}$ (60 neonates in each jar) and fed $6 \mathrm{mg}$ of $R$. subcapitata/L. 
To assess D. magna growth responses to acute contaminant exposure, we sampled 3

164

165 166 167 168 169 170 replicates of 20 neonates placed in pre-weighed aluminum cups and dried overnight at $40^{\circ} \mathrm{C}$ in a drying oven (Fisher Scientific Isotemp Oven) to determine the average initial mass of the daphnids with a microbalance (Sartorius ME 36S, Gorttingen, Germany). After 48 h of growth, differently exposed daphnids were collected from replicate jars ( $n=5$ per treatment) containing 18 D. magna placed in pre-weighed aluminum cups and dried overnight at $40^{\circ} \mathrm{C}$ in a drying oven and reweighed. Mass specific growth rate (MSGR) determined by the equation:

$$
M S G R=\frac{[\ln (B 2)-\ln (B 1)]}{\text { time }}
$$

Where $\mathrm{B} 2$ is the final average mass per D. magna, $\mathrm{B} 1$ is the average initial neonate mass, and time is number of days of D. magna growth. Using mass specific growth rates, instead of mass or length accounts for the small variation in neonate initial size $(1-2 \mu \mathrm{g})$ [43]. MSGR ranges between $0-1$, with 1 indicating a doubling of mass per day, and 0 indicating no growth $[20,43]$.

\section{Adult specific experimental design}

Fresh stock contaminants in the nominal concentration were prepared $(0.67 \mathrm{mg} / \mathrm{L}$ propranolol, $10 \mathrm{mg} / \mathrm{L}$ atrazine, $36 \mathrm{mg} / \mathrm{L} \mathrm{PFOS}$ ) in de-chlorinated tap water. An aliquot of the contaminant was added to replicate $(n=10$ per treatment) glass jars which were subsequently filled to $300 \mathrm{~mL}$ with a $50 \%$ mixture of old and new de-chlorinated tap water to ensure adult survival. This procedure was repeated for all the treatments (propranolol, atrazine, and PFOS). The control treatments used for the propranolol and PFOS exposures, had replicate $(n=10)$ glass jars filled with $300 \mathrm{~mL}$ of a $50 \%$ mixture of old and new de-chlorinated tap water. Atrazine replicate solvent controls $(n=10)$ were prepared with $300 \mathrm{~mL}$ of a $50 \%$ mixture of old and new 
186

187

de-chlorinated tap water containing $0.03 \%$ acetone. We added day 18 old D. magna (from day 0 to 18 grown under D. magna culturing conditions) to each replicate at the density of 1 adult per $30 \mathrm{~mL}$ ( 10 adults per jar) and they were fed $6 \mathrm{mg}$ of $R$. subcapitata $/ \mathrm{L}$ at the start of the experiment and after $24 \mathrm{~h}$.

\section{Animal preservation for ${ }^{1} H$ NMR metabolomics}

After $48 \mathrm{~h}$ of sub-lethal contaminant exposure, we transferred replicate jars to dechlorinated tap water to remove algae and contaminants from their carapace. After rinsing, animals were filtered onto a mesh, transferred to a $1.5 \mathrm{~mL}$ microfuge tube and immediately placed in liquid nitrogen. Animals were subsequently lyophilized and stored at $-20^{\circ} \mathrm{C}$ for 2 weeks before metabolomic analysis was performed.

\section{${ }^{1} H$-NMR Metabolomics extraction}

Daphnia magna from replicate jars were weighed on a microbalance (Sartorius ME 36S, Gorttingen, Germany) to approximately $1 \mathrm{mg}$. The number of $D$. magna required per replicate varied between treatments especially for neonates with $60-100$ individuals, whereas only 2 adults were needed to obtain $1 \mathrm{mg}$. For each treatment (control, solvent control, propranolol, atrazine, and PFOS) we extracted 8 replicates from the neonate and 10 replicates from the adult treatments. Extraction procedures follow Nagato et al. [33]. Briefly, daphnids were homogenized into a fine powder with a $5 \mathrm{~mm}$ stainless steel spatula. After homogenization $45 \mu \mathrm{L}$ of $0.2 \mathrm{M}$ phosphate buffered ( $\mathrm{NaH}_{2} \mathrm{PO}_{4} \cdot 2 \mathrm{H}_{2} \mathrm{O} ; 99.3 \%$; Fisher Chemicals) $\mathrm{D}_{2} \mathrm{O}(99.9 \%$ purity, pD 7.4 adjusted with $30 \% w / w$ NaOD 99.5\% purity; Cambridge Isotope Laboratories) containing 0.1 $w / v$ sodium azide (99.5\% purity; Sigma Aldrich) and $10 \mathrm{mg} / \mathrm{L}$ of 2,2-dimethyl-2-silapentane-5- 
sulfonate sodium salt (DSS; 97\%, Sigma Aldrich) was added as internal calibrant. Samples were then vortexed for $30 \mathrm{~s}$ and sonicated for $15 \mathrm{~min}$. After sonication, samples were centrifuged at $14000 \mathrm{RPM}(\sim 15000 \mathrm{~g})$ at $4{ }^{\circ} \mathrm{C}$ for $20 \mathrm{~min}$. The supernatant was then removed and transferred into a $1.7 \mathrm{~mm}$ NMR tube (Norell Inc., NJ, USA).

\section{Data acquisition and processing}

The ${ }^{1} \mathrm{H}$ NMR spectra were acquired with a Bruker BioSpin Avance III $500 \mathrm{MHz}$ NMR equipped with a ${ }^{1} \mathrm{H}-{ }^{13} \mathrm{C}-{ }^{15} \mathrm{~N}$ TXI $1.7 \mathrm{~mm}$ microprobe fitted with an actively shielded $\mathrm{Z}$ gradient [33]. Briefly, acquisition was performed with 256 scans with a relaxation delay of $3 \mathrm{~s}, 32 \mathrm{k}$ time domain points, and a $90^{\circ}$ pulse calibrated on a per sample basis. Water suppression was accomplished through Presaturation Using Relaxation Gradient and Echoes program (PURGE; [44]). All spectra were apodized through multiplication with an exponential decay corresponding to $0.3 \mathrm{~Hz}$ line broadening in the transformed spectra, with a zero filling factor of $2[30,33]$. The resulting NMR spectra were manually phased, baseline corrected and aligned to $\delta=0.00 \mathrm{ppm}$ using the trimethyl silyl group of the DSS internal calibrant.

\section{Data analysis}

Differences in growth rate caused by contaminant exposure were determined using a ttest (two-tailed, equal variance; Excel 2013 with data analysis add on) between control neonates to the propranolol and PFOS exposed neonates. To determine differences in growth for atrazine exposed animals a t-test (two-tailed, equal variance; Excel 2013 with data analysis add on) between solvent control and atrazine neonates were performed. We set the critical $p \leq 0.05$ adjusted with a Bonferroni correction for multiple comparisons $(p \leq 0.0125)$. 
The ${ }^{1} \mathrm{H}$ NMR spectra region from 0.5 to $10 \mathrm{ppm}$ was divided into $0.02 \mathrm{ppm}$ widths resulting in a total of 475 buckets using AMIX software (v3.9.7; Bruker BioSpin Rheinstetten Germany). Residual water peaks residing in the area between $4.70-4.85 \mathrm{ppm}$ were excluded to eliminate differences due to water suppression. All spectra were scaled to total intensity. The resulting intensity of each bucket was used to construct a principal component analysis (PCA) plot with corresponding PCA loadings plots. The PCA scores plot was exported from AMIX into Sigma Plot 12.5 to graph the average PCA scores plot. PCA is a multivariate unsupervised technique that enables the visualization of many response variables at one time. This technique is ideal for non-targeted metabolomics that generates 475 buckets (response variables) from 1 spectra. If no separation is observed between the control and exposed treatments it indicates the metabolome was not altered. However if significant separation is observed then individual metabolites can be identified and examined to determine the response between control and exposed treatments.

Metabolites were identified by comparing the resonances in the spectra obtained to the AMIX database as well as published resonances for selected metabolites $[15,30,33,34]$. The percent change of metabolites was calculated by using the bucket intensities for the exposed groups (atrazine, PFOS, and propranolol) and the control groups (solvent control and control). Ttests (two-tailed, equal variances; Excel 2013 with data analysis add on) were performed for known metabolites between control treatment to the solvent control, PFOS, and propranolol using $p \leq 0.05$ corrected with the false discovery rate [45]. The atrazine treatment was only compared to the solvent control, thus minimizing any metabolomics changes due to the presence of acetone. Additional t-test (two-tail unequal variances; Excel 2013 with data analysis add on) 
were performed on the relative percent changes of the targeted metabolite between neonate and adult $D$. magna using $p \leq 0.05$ corrected with the false discovery rate [45].

\section{RESULTS}

Daphnia magna growth rates were altered depending on the contaminant exposure.

Neonate daphnids in the solvent control (exposed to $0.03 \%$ acetone) and PFOS exposure did not decrease their growth rates compared to the control animals, thus maintaining a growth rate of $\sim 50 \%$ per day (doubling their body mass every two days; Figure 1). However, neonates exposed to atrazine $(t(8)=6.93, p<0.001)$ and propranolol $(t(8)=13.39, p<0.001)$ caused significant decreases in growth compared to the control neonates (Figure 1). The reduction in growth was more severe in propranolol exposure with neonates only growing $\sim 11 \%$ per day, whereas atrazine exposed neonates were able to grow $\sim 30 \%$ per day (Figure 1 ).

The first 2 axes of the average PCA scores plots explained $86 \%$ and $85 \%$ of the variation for the neonate and adult $D$. magna respectively. Average PCA scores plots of the metabolome identified significant separation in the animals exposed to propranolol and PFOS from the controls in both neonate and adult daphnids (Figure 2). The atrazine treatment caused significant separation from the solvent controls (contains $0.03 \%$ acetone) in only the adult daphnids (Figure 2B). There was no significant separation observed in the PCA scores plots between the controls and the solvent controls in both neonate and adult daphnid treatments (Figure 2). Separation along $\mathrm{PC} 1$ for the neonate $D$. magna was caused by resonances related to the metabolites $\operatorname{arginine}(\delta=1.91 \mathrm{ppm})$, valine $(\delta=1.03 \mathrm{ppm})$, and leucine $(\delta=0.95 \mathrm{ppm})$, and separation along the PC2 axis was caused by alanine $(\delta=1.47$ ppm; Figure $3 \mathrm{~A})$. For adult D. magna separation along PC1 was caused by leucine $(\delta=0.95 \mathrm{ppm})$ and overlapping sugars and amino acids 
resonances, while separation along PC2 was caused by alkanoic acids $(\delta=1.27 \mathrm{ppm}$ and $\delta=$ 1.29 ppm; Figure 3B).

No significant changes were observed in targeted metabolites for the solvent control treatment in both neonates and adult D. magna (Supplemental Data, Figure S2). However the 3 contaminants assessed caused unique changes within the targeted metabolites compared to the controls for both neonate and adult D. magna (Figure 4). Significant differences were observed for most targeted metabolites with the exception of threonine, proline, serine and lactate for all 3 contaminant exposures in both neonates and adults (Figure 4 and Supplemental Data, Figure S3). Generally, neonates exposed to propranolol significantly increased many of amino acids metabolites (between $\sim 15-70 \%$ ) and had decreased glucose and tryptophan compared to the control (Figure 4). Neonates exposed to atrazine resulted in only significant increases in alanine (increase $\sim 50 \%$ ) with all other metabolites remaining the same as the solvent control (Figure 4). Finally neonates exposed to PFOS decreased some of their amino acids metabolites (leucine, isoleucine, valine, alanine, arginine, phenylalanine, and tyrosine, $\sim 5-20 \%$ change decrease) compared to the controls where other targeted metabolites did not differ (Figure 4). Adults exposed to propranolol resulted in many amino acids (except glycine) being increased ( 20-70\% increase) and decreased glucose compared to the control group. Atrazine exposure in adult $D$. magna caused a similar metabolic profile to propranolol however glycine was significantly decreased ( $20 \%)$ compared to the solvent controls. Adult exposure to PFOS revealed increases in many amino acid metabolites (except glycine) similar to propranolol, however no decrease in glucose was observed (Figure 3).

Neonate and adult daphnids exposed to propranolol had similar metabolic profiles, with glucose and lysine being the only targeted metabolites identified as significantly different with 
ontogeny (Figure 4). Relative percent metabolite changes for both atrazine and PFOS exposures differed with ontogeny. The distinct metabolic profiles with ontogeny in the atrazine treatment is caused by the neonate profile remaining similar to the control whereas the adult profile was drastically different from the control (Figure 4). Many of the significant metabolic changes produced by ontogeny in animals exposed to PFOS were caused by inverse metabolic responses with neonates decreasing and adults increasing their metabolites (Figure 4)

\section{DISCUSSION}

In agreement with our hypothesis, the metabolic profile in D. magna likely shifted depending on the contaminant MOA and between neonate and adult acute sub-lethal exposures. Each contaminant tested exhibited unique metabolic responses. In addition to metabolic changes due to contaminant MOA, we also identified differences in the metabolic responses between ontogeny for 2 out of 3 contaminants. While propranolol exposures responded similarly between neonate and adult daphnids, both atrazine and PFOS exposures revealed divergent metabolic profiles with ontogeny. Our results indicate the metabolic profile is altered due to contaminant exposure and these changes are not always consist through ontogeny. Furthermore, these results suggest that the ecological impacts of contaminant exposure are not equal across ontogeny in $D$. magna and could alter ecosystem dynamics due to different stressors on various ages.

When organisms are exposed to a different class of contaminants their biochemistry, and thus metabolic pathways, are altered in a specific way $[15,30,34,46]$. The use of metabolomics, which investigates the intermediates of metabolic pathways, is becoming increasingly popular in toxicity studies to help elucidate the toxic MOA of contaminants $[30,34,46]$. However not all metabolic changes are monotonic over a gradient of contaminant exposure [15,34]. Often 
metabolic changes can have a threshold where metabolites plateau after a certain concentration of contaminant, or have a unimodal response where the highest concentration of exposure decreases, compared to the intermediate concentrations [15,34]. Given that the metabolite responses can vary considerable with exposure concentration, it is best to determine the MOA over a range of contaminant concentration to gain fine scale information on how metabolites change [15]. Although the precise MOA cannot be determined from our experimental design, our study provides some insight into the possible mechanisms causing the alterations in the metabolic profiles when D. magna are exposed to contaminant stress.

In invertebrates, exposure to $\beta$-blockers (i.e. propranolol) cause decreases in heart and feeding rates [23]. We found significant decreases in growth with propranolol exposure as well as increases in the relative amount of most amino acids metabolite and decreases in glucose for both the neonate and adult treatments. Taylor et al. [46] identified the toxic MOA of propranolol in D. magna to involve impairments of fatty acid synthesis. The extraction of metabolites with a phosphate buffer used in the present study is more polar than the chloroform methanol water extraction used by Taylor et al. [46], thus many fatty acids such as linoleic acid would not be visible in our method. Although insignificant, Taylor et al. [46] did report increases in most amino acid metabolites, the discrepancy between these studies maybe caused by differences in concentration of propranolol with Taylor et al. [46] using $10 \%$ of the LC50 compared to $28 \%$ of the LC50 in the present study. With the observed decreases in growth rate and glucose, and increases in many amino acid metabolites, we speculate the metabolomic profile of $D$. magna exposed to propranolol may be attributed to protein degradation being used to fuel energetic demands [47]. This general response of increased free amino acids and decreased growth rates is also observed under low food conditions [43], adding evidence that feeding rates may be 
346 decreased when daphnids are exposed to propranolol. Furthermore, increases in aromatic amino 347 acids may indicate impairments to both immune function and nervous system. Tyrosine is a

348 precursor for both melanin formation (L-DOPA) and dopamine synthesis, which is important for 349 immune function [48], and a known neurotransmitters in invertebrates [49] respectively.

The potential toxic MOA for atrazine in invertebrates is acting as an endocrine disruptor 351 and impairing ecdysteroid activity resulting in difficulty molting, impaired embryogenesis [41], 352 and increased male production [50]. We observed contrasting metabolic responses to atrazine 353 exposure in neonate and adult D. magna. While the neonate exposures did cause decreases in 354 growth, the only metabolic response significantly different from the solvent controls was an 355 increase in alanine content. Alternatively, the adult exposure to atrazine caused many of the 356 amino acid metabolites to increase, and glycine and glucose decreased relative to the solvent 357 controls. In agreement with atrazine's toxic MOA an increase in free amino acid metabolites 358 may be caused by impaired hormone synthesis leading to difficultly molting and embryogenesis as found in crayfish [51]. Or alternatively, the increase in many amino acid metabolites may be 360 attributed to protein degradation, as seen in the propranolol results. Additionally, decreases in 361 glycine may be related to oxidative stress as glycine is needed for the production of a well362 known antioxidant glutathione.

363 Unlike atrazine and propranolol, the toxic MOA of PFOS in Daphnia spp. has yet to be 364 determined at the molecular-level. However the toxic MOA has been reported in other 365 invertebrates such as the earthworm, Eisenia fetida [52] and marine mussels [53]. Lankadurai et 366 al. [52] observed a decrease in many metabolites (valine, leucine, arginine, phenylalanine, and 367 lysine, glucose) and concluded the toxic MOA is likely similar to the mammalian MOA that 368 involves fatty acid oxidation and membrane degradation. In the marine green mussel, Perna 
viridis, an oxidative stress response has been identified by increases in superoxide dismutase, and catalase activity and decreases in glutathione when animals are exposed to PFOS [53].

371 Unlike the propranolol and atrazine neonate exposure, PFOS did not cause a reduction in growth, 372 but did alter the metabolic profile. Our study observed similar metabolic responses as 373 earthworms within the neonate PFOS exposure treatment, with many amino acid metabolites 374 being decreased relative to the control treatments. If the toxic MOA in daphnids disrupts 375 membranes, or makes membranes more permeable, the metabolic profile of neonates exposed to 376 PFOS may be experiencing osmotic stress, as similar metabolome patterns were identified with 377 D. magna exposed to salt stress [43]. Alternatively, many of the amino acids that were found to 378 decrease significantly are essential (e.g. leucine, valine, lysine, and phenylalanine; [54]) this 379 might indicate a slight decrease in feeding rates but not enough to cause growth inhibition. 380 However, to our knowledge no studies have examined the physiological responses of PFOS 381 exposure in Daphnia spp. The adult PFOS exposure resulted in many amino acid metabolites (e.g. alanine, isoleucine, leucine, valine, phenylalanine, methionine) increasing relative to the 383 controls. This pattern of increased amino acids may be caused by protein breakdown to generate 384 energy from glucogenic amino acids (e.g. alanine, valine, methionine) [47]. Alternatively, protein breakdown into amino acid metabolites may be caused by oxidative stress [55]; thus 386 adding evidence that PFOS causes oxidative damage in aquatic invertebrates. Our results indicate the metabolic profiles between neonates and adults differed with the 388 same concentration of contaminant for atrazine and PFOS, while similar responses were 389 observed for propranolol. Previous studies have indicated neonates are more sensitive to 390 contaminant exposure [39,40]; however, our metabolomics study does not support this argument. 391 For example neonates acutely exposed to atrazine had very similar metabolic profiles to the 
392 solvent controls, while the adults exposed to atrazine caused many targeted metabolites to 393 increase compared to the solvent control. We suspect exposing neonates to atrazine caused the 394 decreased in growth as seen with other endocrine disruptors in D. magna [56] that allowed for 395 the additional nutrients and energy that would have been required for growth be shuttled into maintenance pools to maintain homoeostasis. However when growth is minimal in adult

397 Daphnia spp., exposure to atrazine may alter metabolic pathways involved in reproduction and 398 maintenance leading to changes in the metabolic profile, consistent with our observation. To

399 further explore this explanation for differences between ontogeny we suggest future experiments 400 growing D. magna under a range of atrazine at various life stages from neonate, juvenile and 401 young adults where growth is still important life history trait. In addition, to tease apart whether 402 reproduction or maintenance demands are altered in adults, we recommend targeted metabolomics to look at reproduction specific pathways such as vitellogenin formation as well as 404 hormones that could further support the proposed MOA. Daphnia magna exposed to PFOS had many targeted metabolites decreased in neonates 406 and increased in adults compared to unexposed animals. We suspect these differences in the 407 metabolic profiles of neonate and adult daphnids could indicate a difference in the toxicity to 408 sensitive to PFOS exposure, it does indicate potential different metabolic pathways are altered 410 between neonate and adult D. magna. These differences in metabolic profiles between neonate 411 and adult daphnids may be caused by differences in the energetic demands for maintenance, 412 growth, and reproduction as predicted by dynamic energy budget theory [37]. Alternatively, 413 differences seen daphnids metabolic profiles during ontogeny may be caused by differences in 414 physiology leading to changes in contaminant exposure. While it is unknown if PFOS uptake in 


3

D. magna occurs by dietary or environmental routes [12] it is possible both routes of uptake may result in neonates being exposed to less contaminant. If dietary exposure is possible, adults would experience higher concentrations of PFOS as their overall food intake is higher than neonates even though neonates have a faster feeding rate [57,58]. Additionally, if uptake of PFOS comes directly from the environment it may decrease the mass specific contaminant concentration in neonates caused by fast growth rates resulting in growth dilution of contaminants [59].

(1)

5

and adult D. magna. Dissimilar metabolic profiles between ages in D. magna exposed to contaminants may be caused by differences physiology with neonates prioritizing growth while adults prioritize reproduction. If the differences in the metabolic profiles during ontogeny indicates altered stress responses, it may lead to changes daphnids population structure in natural ecosystems by favoring neonates or adults, thus changing foodweb dynamics. Variation in the molecular-level responses throughout ontogeny may add difficultly for the development of high throughput molecular-based techniques for risk assessments as proposed by Schroeder et al. [60]. The goal of examining molecular-level responses including metabolic profiles is to identify changes at the individual level that will correlate to higher level processes such as population growth rates. However, variation in the metabolic profiles caused by ontogeny will likely make it difficult to predict the outcome of contaminant exposure in daphnids. Future experiments should focus on developing models that will be able to predict how the metabolome responds to 
438

439

440 441

442

443

444

445

446

447

448

449

450

451

452

453 1. Wilson HF, Xenopoulos MA. 2008. Effects of agricultural land use on the composition of

454

455

456

457

458

459

460

contaminant exposure throughout ontogeny. Our results indicate care should be taken especially for development molecular-level ecological risk assessments, until the metabolic profile between ontogeny and contaminant stress and their interaction can be predicted.

\section{SUPPLEMENTAL DATA}

Supplemental Data - The Supplemental Data are available on the Wiley Online Library at DOI: XXX

Figures S1-S3

Acknowledgment - We thank the Krembil Foundation for supporting this research. We also thank Vera Kovacevic, Edward Nagato, and Ronald Soong for their assistance with culturing algae and Daphnia as well as NMR data processing. Data Availability. Email ND Wagner at nicolegouldingwagner@gmail.com to request data.

\section{1}

52

\section{REFERENCES} fluvial dissolved organic matter. Nat. Geosci. 2:37-41.

2. Martínez-Bueno MJ, Gomez MJ, Herrera S, Hernando MD, Agüera A, Fernàndez-Alba AR. 2012. Occurrence and persistence of organic emerging contaminants and priority pollutants in five sewage treatment plants of Spain: Two years pilot survey monitoring. Environ. Pollut. 164:267-273.

3. Rivera-Utrilla J, Sánchez-Polo M, Ferro-García MÁ, Prados-Joya G, Ocampo-Pérez R. 2013. Pharmaceuticals as emerging contaminants and their removal from water. A review. 
Chemosphere. 93:1268-1287.

462 4. Heberer T. 2002. Occurrence, fate, and removal of pharmaceutical residues in the aquatic 463 environment: a review of recent research data. Toxicol. Lett. 131:5-17.

464 5. Crosbie B, Chow-Fraser P. 1999. Percentage land use in the watershed determines the water and sediment quality of 22 marshes in the Great Lakes basin. Can. J. Fish. Aquat.

467 6. Graymore M, Stagnitti F, Allinson G. 2001. Impacts of atrazine in aquatic ecosystems. Environ. Int. 26:483-495.

7. Pal A, Gin KYH, Lin AYC, Reinhard M. 2010. Impacts of emerging organic contaminants on freshwater resources: Review of recent occurrences, sources, fate and effects. Sci. Total Environ. 408:6062-6069.

472

8. Mompelat S, Le Bot B, Thomas O. 2009. Occurrence and fate of pharmaceutical products and by-products, from resource to drinking water. Environ. Int. 35:803-814.

474 9. Fleeger JW, Carman KR, Nisbet RM. 2003. Indirect effects of contaminants in aquatic ecosystems. Sci. Total Environ. 317:207-233.

10. Jarvis AL, Bernot MJ, Bernot RJ. 2014. The effects of the psychiatric drug carbamazepine on freshwater invertebrate communities and ecosystem dynamics. Sci. Total Environ.

479 11. González-Pleiter M, Gonzalo S, Rodea-Palomares I, Leganés F, Rosal R, Boltes K, Marco 480 E, Fernández-Piñas F. 2013. Toxicity of five antibiotics and their mixtures towards photosynthetic aquatic organisms: Implications for environmental risk assessment. Water

483 12. Boudreau TM, Sibley PK, Mabury SA, Muir DGC, Solomon KR. 2003. Laboratory 
evaluation of the toxicity of perfluorooctane sulfonate (PFOS) on Selenastrum capricornutum, Chlorella vulgaris, Lemna gibba, Daphnia magna, and Daphnia pulicaria. Arch. Environ. Contam. Toxicol. 44:307-313.

13. Palma P, Palma VL, Fernandes RM, Soares AMVM, Barbosa IR. 2008. Acute toxicity of atrazine, endosulfan sulphate and chlorpyrifos to Vibrio fischeri, Thamnocephalus platyurus and Daphnia magna, relative to their concentrations in surface waters from the Alentejo region of Portugal. Bull. Environ. Contam. Toxicol. 81:485-489.

14. Zhang L, Niu J, Li Y, Wang Y, Sun D. 2013. Evaluating the sub-lethal toxicity of PFOS and PFOA using rotifer Brachionus calyciflorus. Environ. Pollut. 180:34-40.

15. Nagato EG, Simpson AJ, Simpson MJ. 2016. Metabolomics reveals energetic impairments in Daphnia magna exposed to diazinon, malathion and bisphenol-A. Aquat. Toxicol. 170:175-186.

16. Phyu YL, Warne MSJ, Lim RP. 2005. Toxicity and bioavailability of atrazine and molinate to the freshwater shrimp (Paratya australiensis) under laboratory and simulated field conditions. Ecotoxicol. Environ. Saf. 60:113-122.

17. Sole M, Shaw JP, Frickers PE, Readman JW, Hutchinson TH. 2010. Effects on feeding rate and biomarker responses of marine mussels experimentally exposed to propranolol and acetaminophen. Anal. Bioanal. Chem. 396:649-656.

18. Liu Y, Chen T, Li MH, Xu HD, Jia AQ, Zhang JF, Wang JS. 2015. ${ }^{1}$ H NMR based metabolomics approach to study the toxic effects of dichlorvos on goldfish (Carassius auratus). Chemosphere. 138:537-545.

19. Samuelsson LM, Förlin L, Karlsson G, Adolfsson-Erici M, Larsson DGJ. 2006. Using NMR metabolomics to identify responses of an environmental estrogen in blood plasma of 
fish. Aquat. Toxicol. 78:341-349.

508

509

510

511

512

513

514

515

516

517

518

519

520

521

522

523

524

525

526

527

528

529

20. Sterner R, Elser J. 2002. Ecological stoichiometry: The biology of elements from molecules to the biosphere.

21. Elser JJ, Chrzanowski TH, Sterner RW, Mills KH. 1998. Stoichiometric constraints on food-web dynamics: A whole-lake experiment on the Canadian Shield. Ecosystems. $1: 120-136$.

22. Prater C, Wagner ND, Frost PC. 2015. Effects of calcium and phosphorus limitation on the nutritional ecophysiology of D aphnia. Limnol. Oceanogr.: 61:268-278.

23. Dzialowski EM, Turner PK, Brooks BW. 2006. Physiological and reproductive effects of beta adrenergic receptor antagonists in Daphnia magna. Arch. Environ. Contam. Toxicol. 50:503-510.

24. Ji K, Kim Y, Oh S, Ahn B, Jo H, Choi K. 2008. Toxicity of perfluorooctane sulfonic acid and perfluorooctanoic acid on freshwater macroinvertebrates (Daphnia magna and Moina macrocopa) and fish (Oryzias latipes). Environ. Toxicol. Chem. 27:2159-68.

25. Nikkilä A, Paulsson M, Almgren K, Blanck H, Kukkonen J V. 2001. Atrazine uptake, elimination, and bioconcentration by periphyton communities and Daphnia magna: effects of dissolved organic carbon. Environ. Toxicol. Chem. 20:1003-1011.

26. Andersen T, Elser JJ, Hessen DO. 2004. Stoichiometry and population dynamics. Ecol. Lett. 7:884-900.

27. Cleuvers M. 2005. Initial risk assessment for three $\beta$-blockers found in the aquatic environment. Chemosphere. 59:199-205.

28. Phyu Yin L, Warne MSJ, Lim RP. 2004. Toxicity of atrazine and molinate to the cladoceran Daphnia carinata and the effect of river water and bottom sediment on their 
bioavailability. Arch. Environ. Contam. Toxicol. 46:308-315.

531 29. Garcia-Reyero N, Poynton HC, Kennedy AJ, Guan X, Escalon BL, Chang B, Varshavsky

30. Lankadurai BP, Wolfe DM, Simpson AJ, Simpson MJ. 2011. ${ }^{1}$ H NMR-based metabolomic observation of a two-phased toxic mode of action in Eisenia fetida after sub-

31. Viant MR, Pincetich CA, Tjeerdema RS. 2006. Metabolic effects of dinoseb, diazinon and esfenvalerate in eyed eggs and alevins of Chinook salmon (Oncorhynchus tshawytscha) determined by ${ }^{1}$ H NMR metabolomics. Aquat. Toxicol. 77:359-371.

32. Hughes SL, Bundy JG, Want EJ, Kille P, Stürzenbaum SR. 2009. The metabolomic responses of Caenorhabditis elegans to cadmium are largely independent of metallothionein status, but dominated by changes in cystathionine and phytochelatins. $J$. Proteome Res. 8:3512-3519.

33. Nagato EG, Lankadurai BP, Soong R, Simpson AJ, Simpson MJ. 2015. Development of an NMR microprobe procedure for high-throughput environmental metabolomics of Daphnia magna. Magn. Reson. Chem.:745-753. doi:10.1002/mrc.4236.

34. Kovacevic V, Simpson AJ, Simpson MJ. 2016. ${ }^{1} \mathrm{H}$ NMR-based metabolomics of Daphnia magna responses after sub-lethal exposure to triclosan, carbamazepine and ibuprofen. Comp. Biochem. Physiol. Part D Genomics Proteomics. doi:10.1016/j.cbd.2016.01.004.

35. Taylor NS, Weber RJM, Southam AD, Payne TG, Hrydziuszko O, Arvanitis TN, Viant MR. 2009. A new approach to toxicity testing in Daphnia magna: Application of high 
throughput FT-ICR mass spectrometry metabolomics. Metabolomics. 5:44-58.

554 36. Vandenbrouck T, Jones OAH, Dom N, Griffin JL, De Coen W. 2010. Mixtures of similarly acting compounds in Daphnia magna: From gene to metabolite and beyond. Environ. Int. 36:254-268.

557 37. Kooijman SALM. 2001. Quantitative aspects of metabolic organization: a discussion of 558 concepts. Philos. Trans. R. Soc. B Biol. Sci. 356:331-349.

559 38. Bradley MC, Baird DJ, Calow P. 1991. Mechanisms of energy allocation to reproduction 560 in the cladoceran Daphnia magna Straus. Biol. J. Linn. Soc. 44:325-333.

561 39. Ortiz-Rodriguez R, Wiegand C. 2010. Age related acute effects of microcystin-LR on Daphnia magna biotransformation and oxidative stress. Toxicon. 56:1342-1349.

563

564 565 566 567 568 569 570 571 572 573 574 575

40. Stuhlbacher A, Bradley MC, Naylor C, Calow P. 1993. Variation in the development of cadmium resistance in Daphnia magna straus; Effect of temperature, nutrition, age and genotype. Environ. Pollut. 80:153-158.

41. Palma P, Palma VL, Matos C, Fernandes RM, Bohn A, Soares AMVM, Barbosa IR. 2009. Effects of atrazine and endosulfan sulphate on the ecdysteroid system of Daphnia magna. Chemosphere. 74:676-681.

42. Environment Canada. 2000. Biological test method: Reference method for determining acute leathality of effluents to Daphnia magna.

43. Wagner ND, Lankadurai BP, Simpson MJ, Simpson AJ, Frost PC. 2015. Metabolomic differentiation of nutritional stress in an aquatic invertebrate. Physiol. Biochem. Zool. $88: 43-52$.

44. Simpson AJ, Brown SA. 2005. Purge NMR: Effective and easy solvent suppression. J. Magn. Reson. 175:340-346. 
576 45. Benjamini Y, Hochberg Y. 1995. Controlling the False Discovery Rate : a Practical and 577 Powerful Approach to Multiple Testing. J. R. Stat. Soc. Ser. B. 57:289-300.

578 46. Taylor NS, Weber RJM, White TA, Viant MR. 2010. Discriminating between different 579 580 acute chemical toxicities via changes in the daphnid metabolome. Toxicol. Sci. 118:307317.

581 47. Gillis T, Ballantyne J. 1996. The effects of starvation on plasma free amino acid and 582 glucose concentrations in lake sturgeon. J. Fish. 49:1306-1316.

48. Mucklow PT, Ebert D. 2003. Physiology of immunity in the water flea Daphnia magna: Environmental and genetic aspects of phenoloxidase activity. Physiol. Biochem. Zool. 76:836-482.

586

587

588

589

590

591

592

593
49. Campos B, Garcia-Reyero N, Rivetti C, Escalon L, Habib T, Tauler R, Tsakovski S, Pina B, Barata C. 2013. Identification of metabolic pathways in Daphnia magna explaining hormetic effects of selective serotonin reuptake inhibitors and 4-Nonylphenol using transcriptomic and phenotypic responses. Environ. Sci. Technol. 47:9434-9443.

50. Dodson SI, Merritt CM, Shannahan JP, Shults CM. 1999. Low exposure concentrations of atrazine increase male production in Daphnia pulicaria. Environ. Toxicol. Chem. 18:1568-1573.

51. Mcwhinnie MA, Kirchenberg RJ, Urbanski RJ, Schwarz JE. 1972. Crustecdysone mediated changes in crayfish. Integr. Comp. Biol. 12:357-372.

52. Lankadurai BP, Simpson AJ, Simpson MJ. 2012. ${ }^{1} \mathrm{H}$ NMR metabolomics of Eisenia fetida responses after sub-lethal exposure to perfluorooctanoic acid and perfluorooctane sulfonate. Environ. Chem. 9:502-511.

53. Liu C, Chang VWC, Gin KYH. 2014. Oxidative toxicity of perfluorinated chemicals in 
green mussel and bioaccumulation factor dependent quantitative structure-activity relationship. Environ. Toxicol. Chem. 33:2323-2332.

54. Cowey C, Forster J. 1971. The essential amino-acid requirements of the prawn Palaemon serratus. The growth of prans on diets containing proteins of different amino-acid composition. Mar. Biol. 10:77-81.

55. Valavanidis A, Vlahogianni T, Dassenakis M, Scoullos M. 2006. Molecular biomarkers of oxidative stress in aquatic organisms in relation to toxic environmental pollutants. Ecotoxicol. Environ. Saf. 64:178-189.

56. Leblanc G. Mclachlan JB. 1999. Molt-independent growth inhibition of Daphnia magna by a vertebrate antiandrogen. Environ. Toxicol. Chem. 18:1450-1455.

57. Vanni MJ, Lampert W. 1992. Food quality effects on life history traits and fitness in the generalist herbivore Daphnia. Oecologia. 92:48-57.

58. Brown JH, Gillooly JF, Allen AP, Savage VM, West GB. 2004. Toward a metabolic theory of ecology. Ecology. 85:1771-1789.

59. Karimi R, Chen CY, Pickhardt PC, Fisher NS, Folt CL. 2007. Stoichiometric controls of mercury dilution by growth. Proc. Natl. Acad. Sci. U. S. A. 104:7477-7482.

60. Schroeder AL, Ankley GT, Keith AH, L.Villeneuve D. 2016. Environmental surveillnce and monitoring - the next frontiers for high-throughput toxicology. Environ. Toxicol. Chem. 35:513-525. 


\section{Figure Legends}

620 Figure 1. Average \pm standard error of mass specific growth rate per day $\left(\mathrm{MSGR} \mathrm{d}^{-1}\right)$ of neonate 621 D. magna exposed for $48 \mathrm{~h}$ of propranolol (Pro; $0.67 \mathrm{mg} / \mathrm{L})$, atrazine (10 mg/L), and PFOS (36 $622 \mathrm{mg} / \mathrm{L})$ and unexposed control and solvent control ( $0.03 \%$ acetone; $n=5$ per treatment $)$ * 623 indicate $p \leq 0.05$ corrected for multiple comparisons $(p \leq 0.0125)$ between control and exposed 624 treatments.

Figure 2. Average principal component analysis (PCA) score plots \pm standard error $(n=8$ per treatment for neonate; $n=10$ per treatment for adult) for: (A) neonate and (B) adult D. magna grown for $48 \mathrm{~h}$ under control, and solvent conditions and exposed to propranolol $(0.67 \mathrm{mg} / \mathrm{L})$, atrazine $(10 \mathrm{mg} / \mathrm{L})$, and PFOS (36 mg/L). * indicate $p \leq 0.05$ corrected for multiple comparisons $(p \leq 0.0125)$ between control and exposed treatments.

631

Figure 3. Principal component analysis (PCA) loading plots indicating buckets responsible for the separation within PCA. (A) PCA loadings for neonate PCA, (B) PCA loadings for adult PCA.

Figure 4. Average relative percent changes \pm standard error of targeted metabolites from the in neonate (grey bars) and adult (white bars) treatments. * indicates significant differences from the control $p \leq 0.05$ corrected for the false discovery rate. + indicates significant differences in the response between neonate and adult treatments relative to their respective controls $p \leq 0.05$ corrected for the false discovery rate. Bolded amino acid names are classified as essential in 
641 crustaceans [54]. Nonsignificant metabolite changes are shown in Supplemental Data, Figure 642 S3. 